\title{
Monitoring a Herd of Pastured Cattle Using Mobile Sensor Networks
}

\author{
Geunho Lee ${ }^{\mathrm{a},},{ }^{*}, K$ ouki Ogata ${ }^{\mathrm{a}}$, Kota Okabe $^{\mathrm{a}}$, Ryoichi Aizawa ${ }^{\mathrm{a}}$, Seiya Sakaguchi ${ }^{\mathrm{a}}$ \\ ${ }^{a}$ University of Miyazaki, 1-1 Gakuen-Kibanadai Nishi, Miyazaki 8892192, Japan \\ *Corresponding Author: geunho@cc.miyazaki-u.ac.jp
}

\begin{abstract}
In this paper, we tackle a monitoring problem for a head of pastured cattle by employing sensors that self-organize their networks while adapting to topological changes. Our challenge is exploiting locally communicative interactions of a relative received signal strength under minimal conditions, such as locality, state memory, and implicit coordination. The solution approach focuses on achieving some network redundancy by selecting specific neighboring sensors with high connectivity, resulting in the determination of favorite relations in a cattle herd. The solution is verified by performing extensive simulations featuring self-organization, topological adaptation, and self-healing. Moreover, the effectiveness of the self-organization is demonstrated in a Miyazaki ranch by using five cows attached to sensor tags. Our approach is effective for the secure adaptive selforganization of mobile sensor networks in real-world sensor network applications.
\end{abstract}

Keywords: herd of cattle, behavior monitoring, mobile sensor network, self-organization.

\section{Introduction}

With advances in mobile network-enabled sensors, considerable effort has been made to increase sensor network applications [1][2]. Several studies employing large-scale mobile sensors have been reported in the fields of network and swarm sensors [3]-[5]. From the viewpoint of the application domain of surveillance or environmental monitoring, large numbers of mobile sensors can be considered as swarms of wireless sensors mounted on spatially distributed autonomous sensors [3][4]. Mobile sensors may be widely deployed across an interesting area with relatively high continuous density. They continue to move until the completion of a desired network configuration. Despite achieving the desired configuration, the network topology is easily affected by movements according to needs or even failures. Meanwhile, if a certain number of sensors send data at exactly the same time, the network might suffer from mutual interference. Sensor networks with such latent aspects remain one of the several issues that need to be addressed. First, mobile sensors need to self-organize their networks while generating their communication paths. Second, it is necessary to partially reflect rather than regenerate topological changes and to quickly restore networks for several sensor failures. Third, developing communicative frameworks with relatively minimal conditions from the mobile point of view is

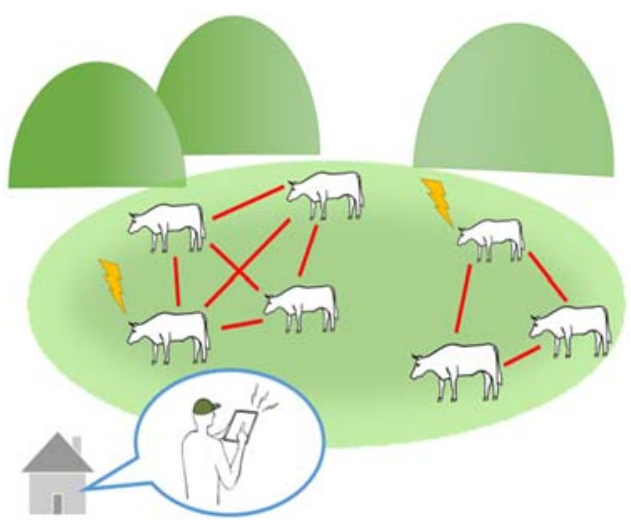

Fig. 1. Concept of monitoring a herd of pastured cattle using mobile sensor networks.

desirable [5].

For the purpose, this paper tackles a self-organized monitoring problem for a head of pastured cattle by employing sensors (see Fig. 1) that self-organize their networks while adapting to topological changes. To solve the addressed problem, our challenge is exploiting locally communicative interactions of a relative received signal strength under minimal conditions, such as locality, state memory, and implicit coordination from a computational standpoint. Based on this minimal-condition model, we propose a high-degree connectivity-based swarm 
communication framework (HCSCF) that enables individual sensors to self-organize their networks while adapting to topological changes according to movements and/or failures. Under HCSCF, each sensor obtains local distribution information for adjacent sensors, selects specific neighbors with high connectivity, and organizes the sensor's locally partial network with a star topology. When local networks overlap, an overall network with a partially connected mesh topology can be generated. Specifically, information obtained by broadcasting and overhearing helps each sensor partially update and adapt to any change in its local network, resulting in the determination of favorite relations in a cattle herd. We describe the proposed HCSCF in detail and perform extensive simulations to demonstrate its self-organization, topological adaptation, and self-healing capabilities. Notably, from a practical point of view, mobile sensor swarms based on HCSCF can self-organize while adapting to topological changes. Through extensive evaluations, it is confirmed that the framework allows mobile sensors to cover an interesting area as sensor networks.

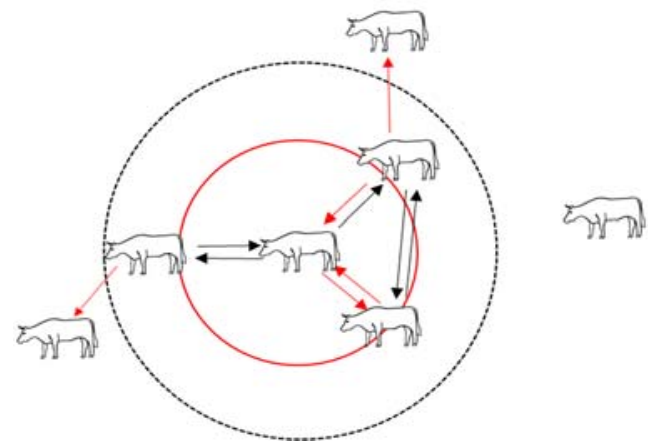

Fig. 2. Self-organized network by gathering local networks with star topologies.

\section{Problem Definition}

We formally tackle the adaptive self-organization problem for a swarm of $n$ autonomous sensors on the basis of the aforementioned model definitions as follows: Given a swarm of sensors above, how can individual sensors with minimal capabilities self-organize their networks while adapting to topological changes in a decentralized way?

This problem seeks a decentralized solution based only on local broadcasting. Individual sensors build their local networks from the distribution of adjacent sensors while removing some redundant communication links. As illustrated in Fig. 2, collection of these local networks also allows the sensors to self-organize the overall network. Although the networks are initially generated, this network is highly changeable by sensor movements and sensor disappearances caused by sensor failures. Therefore, sensors partially update the network configuration according to their changing conditions. Consequently, we advocate that the addressed problem can be addressed by a solution with selforganization, network adaptation, and self-healing capabilities.

\section{Algorithm Description}

This section describes the proposed solution, HCSCF, to the stated problem. $\mathrm{HCSCF}$ is composed of three sequential procedures: 1) information acquisition about the

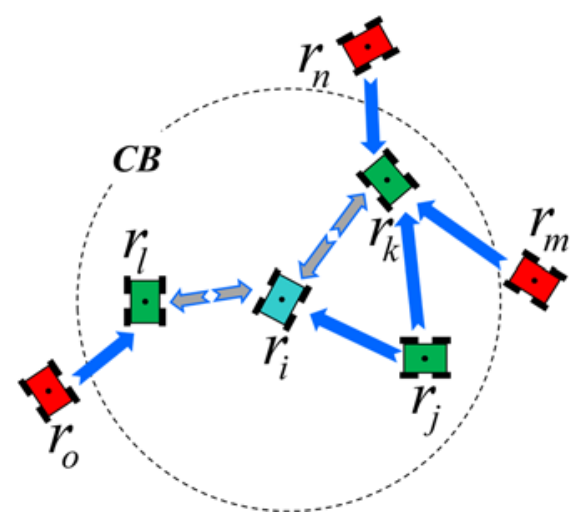

Fig. 3. Illustration of collecting $G_{i}$ as partiallyconnected mesh network.

local distribution of adjacent sensors, 2) neighbor selection computation based on the acquired information, and 3) local network generation.

The first step in HCSCF is to investigate the local configuration of the sensors adjacent to a mobile sensor $r_{i}$ by broadcasting and receiving, including overhearing. The input of the first computation step is messages within the communication range of $r_{i}$. Its outputs are the 1-hop communication sensors $C_{i}^{1}$ and the 2-hop communication sensors $C_{i}^{2}$ with respect to $r_{i}$. Even though these outputs are still local, $r_{i}$ can estimate a more extended network configured by $C_{i}^{1}$ and $C_{i}^{2}$. Ultimately, from the sensor configuration in $C_{i}^{2}, r_{i}$ can count the number of communication links between the sensors and assess their topology configuration. In the second computation, the input arguments of $r_{i}$ are $C_{i}^{1}$ and $C_{i}^{2}$, and its output is sensors $N_{i}$ with high-degree connectivity. As the third computation step, the input of $r_{i}$ is $N_{i}$, and its output is the undirected graph $G_{i}=\left\{V_{i}, E_{i}\right\}$, where $V_{i}$ is a set of $n$ vertices $\left\{v_{1}, v_{2}, \cdots v_{n}\right\}$ and $E_{i}$ is a set of edges between vertices $E_{i}$ 
$=\left\{\left(v_{i}, v_{j}\right) \mid v_{i}, v_{j} \in V_{i}\right\} . \quad$ By collecting $G_{i}=\left\{V_{i}, E_{i}\right\}, \boldsymbol{G}$ can globally self-organize without a centralized control scheme. When the local star networks overlap, the overall network can have the partially connected mesh network topology illustrated in Fig. 3. From the standpoint of network topology, HCSCF enables a sensor to take advantage of some of the network redundancy by connecting to as many adjacent sensors as possible. In particular, if individual sensors agree on the mutual neighbor, $\boldsymbol{E}$ will become a central communication path that is connected to their many adjacent sensors. Details on this algorithm will be introduced in our final submission.

\section{Evaluation Results and Discussion}

This section describes the simulation we conducted to evaluate the validity and effectiveness of HCSCF. Timevarying communication delays were set in these simulation studies. Our in-house simulator was developed in the Microsoft Visual Studio 2019 software development environment to realize sensor movements considering collision avoidance and to represent graphical effects, such as neighbor selection. The simulations were performed by running the simulator under Microsoft Windows 10 Pro. In our simulator, communication buffers for individual sensors were set to implement random communication delays. When a sensor received any broadcasted data, the delays were
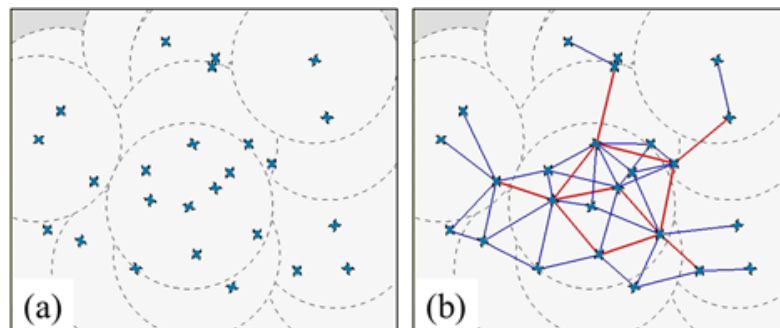

(b)
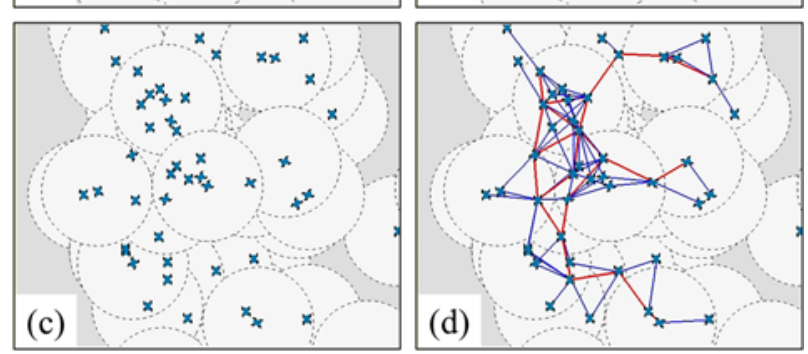

Fig. 4. Simulation results for network organization ((a) initial distribution of 25 robots, (b) sensor network generation, (c) initial distribution of 50 robots, (d) sensor network generation).

randomly generated in the buffer within ten steps. In the simulations, each sensor autonomously moved in a two- dimensional plane.

To evaluate the validity and effectiveness of HCSCF, we performed a series of simulations demonstrating the
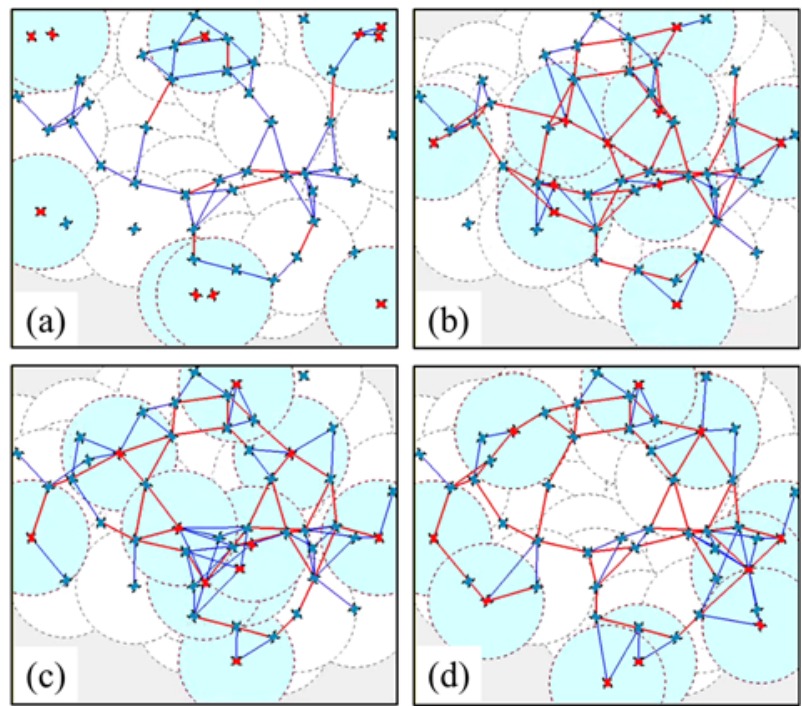

Fig. 5. Simulation result for topological adaptation where 10 red robots move arbitrarily and simultaneously and 40 blue robots remain stationary.

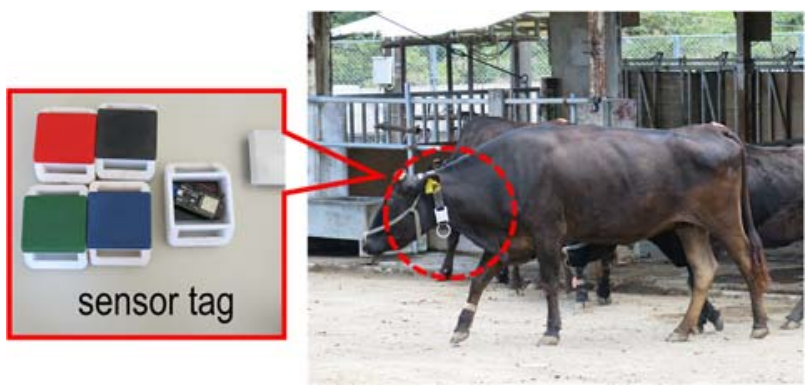

Fig. 6. Experimental setting conducted in Miyazaki cattle ranch where sensor tags attached to the neck of individual cow.

framework's self-organization, topological adaptation, and self-healing features. Fig. 4 shows the simulation results for network generation by 25 and 50 sensors. In Figs. 4-(b) and (d), the blue lines mean the defined edges from $r_{i}$. The red boldfaced lines indicate individual sensors that agreed on the mutual neighbor after the network generation. The sensors could organize their overall mesh network $\boldsymbol{G}$ by collecting local networks $G_{i}$. Fig. 5 presents the simulation results for topological adaptation by 50 sensors. Ten red sensors moved arbitrarily and simultaneously to make topological changes in the generated $\boldsymbol{G}$, but the other sensors remained stationary. Under HCSCF, the sensors partially updated $C_{i}^{1}, C_{i}^{2}$, and $N_{i}$ by overhearing; they also modified a part of $\boldsymbol{G}$ corresponding to the changes rather than performing overall 


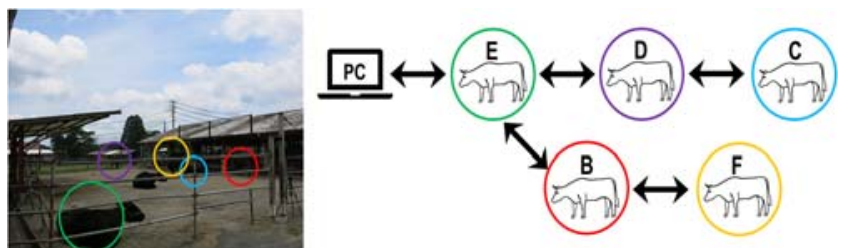

(a)

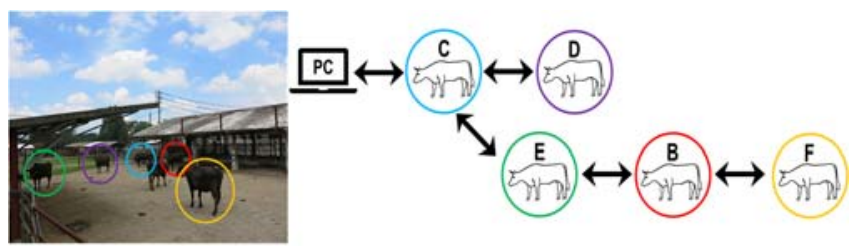

(b)

Fig. 7. Experimental result for topological adaptation with respect to $P C$ according to movements of five sensors (according to snapshots taken at (a) 11:42, (b) 13:14).

regeneration. The result showed that $G_{i}$ could adapt to topological changes according to the sensor movements around $r_{i}$. Therefore, the sensor swarms could self-organize themselves while adapting to network changes.

Next, experiments were conducted to verify that sensor swarms can self-organize themselves while adapting to topological changes. To play a role as mobile sensors, the number of five cows in the Miyazaki cattle ranch was selected. Moreover, the five cows were attached to sensors tags. Fig. 6 shows the snapshots of sensor tags and the scene where a cow wore a neck belt with a sensor tag. In practice, experiments for topological adaptation in the sensor network composed of five mobile sensors equipped with Espressif ESP-WROOM-32D tags were conducted. In this experiment as shown in Fig, 7, we examined the topological adaptation of a fixed sensor tag $P C$ when five sensors move in the swarm. The left side in Fig. 7 indicates experimental scenes and the right side is states of the $P C$ 's local network configuration as time went on. From the experiments, we confirmed that HCSCF and its realization on these tags mounted on cows could be considered quite satisfactory for the practical use of mobile sensor networks.

\section{Conclusions}

In this paper, the self-organized monitoring problem for a cattle herd was tackled to organize an overall network that adapts to topological changes. As our decentralized solution, HCSCF was proposed, which allowed sensors with minimal capabilities to identify neighbors with high connectivity. Under HCSCF, the local networks of sensors could be generated with neighbor-based star topologies. When collecting such local networks, sensor swarms could self-organize a global network with partially connected mesh topologies. The proposed algorithm also enabled sensors to self-adapt their local networks to topological changes by sensor movements and failures. Moreover, the neighbor selection method provided positive possibilities for mutual interference, channeling communication paths, and network redundancy. To demonstrate the validity and effectiveness of HCSCF, extensive simulations and experiments by using pastured cows were performed, and the results were analyzed. The results were quite encouraging, and we confirm that these will provide autonomous sensor swarms with better possibilities toward mobile sensor networks. In our future work, we will attempt to study connectivity enhancement issues in mobile sensor networks based on HCSCF.

\section{Acknowledgment}

This work is supported by the Research Grant awarded by the Telecommunications Advancement Foundation.

\section{References}

(1) J. K. Park and E. Y. Park, "Monitoring method of movement of grazing cows using cloud-based system", ECTI Transactions on Computer and Information Technology, Vol.15, No.1, pp.24-33, 2021

(2) F. Zhou, R. Q. Hu, Z. Li, and Y. Wang, "Mobile edge computing in unmanned aerial vehicle networks", IEEE Wireless Communications, Vol.27, No.1, pp.140-146, 2020

(3) S. Muhammad, N. Mohammad, A. Bashar, and M.A. Khan: "Designing human assisted wireless sensor and robot networks using probabilistic model checking", Journal of Intelligent and Robotic Systems, Vol.94, pp. 687-709, 2019

(4) R. Kazala, A. Taneva, M. Petrov, S. Penkov: "Wireless network for mobile robot applications", IFACPapersOnLine, Vol.48, pp.231-236, 2015

(5) H. C. H. Chiu and W.-M. Shen, "ANCHOR - selfconfiguring robotic network", Proceedings of the IEEE International Conference on Robotics and Automation, pp.5375-5380, 2011 